\title{
Zweite Abtheilung.
}

\section{Vereins - Zeitung, \\ redigirt vom Directorium des-Vereins.}

\section{Ein 50jähriges Apotheker-Jubilăum in Russland.}

Es sind nun mehr als 150 Jahre, seitdem Peter der Grosse das Privilegium zur ersten freien Apotheke in Moskau (1701) und dann für St. Petersburg (1706) Höchsteigenhändig unterzeichnet hat. Seit dieser Zeit entwickelte sich immer mehr das pharmaceutische Studium in Russland; eine Reihe ausgezeichneter Apotheker bekundeten, dass die Pharmacie mit den übrigen Wissenschaften bei uns gleichen Schritt gehalten; ein Model, ein Lowitz, ein Scherer und mehrere noch mit uns lebende Männer der pharmaceutischen Wissensehaften haben weit über die Grenzen Russlands hinsus ihrem Namen eine europäische Geltung zu verschaffen gewusst. Während dieses ganzen Zeitraumes war bis jetzt nur einem Einzigen das Glïck zu Theil geworden, sein 50jähriges ApothekerJubiläum zu feiern; es musste demnach bei allen Standesgenossen, und besonders bei den Mitgliedern der pharmaceutischen Gesellschaft in St. Petersburg, den innigsten Anklang erregen, dass abermals mit hoher Erlaubnias der Regierung, ein 50 jähriges A pothekerJubiläum durch eine solenne Feier begangen werden soll.

Diese bei uns so seltene Festfeier galt diesmal dem in der Wissenschaft und im Leben viel geachteten und geliebten Manne, dem Ober-Berg-Apotheker Alex ander Kämmerer, der heute vor 50 Jahren sein erstes Diplom als Pharmaceut sich erwarb.

Nicht Jedem ist es genug bekannt, was es bedeuten will, als Apotheker ein halbes Jahrhundert hindurch gearbeitet, gewirkt und genützt zu haben, und trotz diesen Mühen, diesen Sorgen, den oft so schädlichen Einflüssen bei den pharmaceutischen Arbeiten, dennoch, so wie unser Jubilar, geistig und körperlich so frisch und gesund geblieben zu sein. Bescheiden und anspruchslos ist die Thïtigkeit des wissenschaftlich gebildeten Apothekers; nur die grösste Gewissenhaftigkeit ist der Grundpfeiler seines Wirkcns, und so verfliesst sein ganzes Leben in stiller Arbeit, im Bewusstsein erfüllter Pflichten. Wenig Ermunterung, geringe Anerkennung, und die Masse glaubt, dass durch seine materiell günstigeren Verhältnisse, die so oft nur scheinbar sind, ihm in Allem Genüge geschehen sei.

Um so mehr nun mass das Horz eines fuhlenden Menschen hoch erfreut werden, wenn er sieht, wie der Lohn der rüstigen Arbeit folgt, wie Anerkennung dem muthigen Streben zur Seite

Arch. d. Pliarm. CXXXXI. Bds. 3. Hft. 
geht. wie das Verdienst seine gerechte Würdigung findet. Cond so geschah es bis zum heutigen Festtage mit unserm geachteten Jubilar! Er war vor 39 Jahren Mitstifter der durch ihr naturwissenschaftliches Museum jetzt ausgezeichneten, blühenden plarmaceutischen GesellBchaft, ist seit 15 Jahren ihr thätiger Director und seit heute Ehrenmitglied Das in Goldsehrift ausgefertigte I'rachtdiplon erregte die Bewunderung aller Anwesenden.

Der in Gott ruhende Kaiser Nicola i würdigte schon die Beruf'streue Kärn merer's durch Anszeichnungen jeder Art, anch ernannte er ihn zum Mitlehrer bei seiuen Kaiserlichen Kindern. Lnser jetziger erhabener Herr und Kaiser, grossherziger Beschützer und Förderer jeglichen wissenschaftlichen und gemeinnützigen Strebens, ernannte Kämmerer am Jubiläumstage zum wirklichen Staatsrath, einem Range, der bis jetzt noch keinem Apotheker zu Theil ge:worden ist.

In der Mineralogie ist es bekannt, was der Name $K$ änmerer gilt, und gewiss wohlverdient wurde ihm zu Ehren ein zu Bissersk in Sibirien neu entdecktes Mineral (das aus Kiesel -, Thon- und Talkerde, Chrom und Eisenoxydul besteht) vor mehreren Jahren von den Männern der Wissenschaft Kämmererit genannt. Wir wollen uns nicht bei allen Fest- und Gratulationsgeschenken für den Jubilar aufhalten, den bereits 13 gelehrte Corporationen des In- und Auslandes zu ihrem Mitgliede rechnen*).

Eilen wir zur Hauptfeier des Tages, zum Festesben, das am Mittwoch in der Osterwoche im Saale der pharmaceutischen Gesellschaft statt fand.

Beim Eintritt in den Saal wurde der geehrte Jubilar, anf dessen Antlitze frische Gesundheit und freundlichstes Wohlwollen, wie immer, sich aussprach, unter Begleitung einer Festcantate, von sämmtlichen Mitgliedern der pharmaceutischen Gesellschaft bewillkommnet, beglück ẅ̈nscht und umarmt. Der schön erleuchtete Saal war festlich geordnet und das Portrait des Jubilars an der Wand, in der Mitte der Portraits der früheren Directoren der Gesellschaft, nit Lorbeerkrïnzen geschmückt. Die Tafel, mit Blumen geziert, genügte vollkommen so dem Feinschmecker wie dem Feintrinker.

Der erste Toast galt, wie die gute Sitte es verlangt, Sr. Maje. stät dem Kaiser und dem ganzen hohen Kaiscrhause, wobei das zahlreiche Orchester die Nationalhymne spielte. Nun erhob sich der um die Gesellschaft hochverdiente, 30 Jahre schon fungirende Secretair derselben, Herr Apotheker Pfeffer, und hielt cinen ausführlichen 'Tischspruch auf den Jubilar, worin er besonders dessen Verdienste un die Gesellsehaft, um den ganzen pharmaceutischen Stand in sehr gewählten Worten hervorhob. Am Schlusse seines sehr ansprechenden Vortrages prïsentirte der Redner im Narnen der Apotheker St. Petersburgs und einigrer des Inlandes einen kost. baren, mit sinnreicher lateinischer Inschrift versebenen Pokal, der, ganz mit schäunendem Champagner gefüllt, unter drcimaligem, die Musik übertïnendem Hoch! zuerst vom Jubilar an die Lippen gesetzt wurde und dann, indem sehr fleissig nachgegossen wurde, die

*) Das Directorium des deutschen Gesammt-Apotheker-Vereins sindte dem trefflichen Jubilar das Fhrendiplom, so wie der Oberdirector Dr. Bley den ersten Band des Archivs der Pharmacie, Jahrgang 1857, in schönem lrachtbando, mit cinem freundliehen Schreiben ein. 
Runde um den ganzen Tisch machte. Den Dankesworten des tief gerührten Jubilars folgte sofort ein Toast auf Se. Excellenz den allverebrten Herru Minister des Innern, der der Gesellsehaft und ihrem Director oft schon seine wohlwollende Zuneigung kund gegeben hat. Nachdem noch auf das Wobl der Gäste getrunken worden war, bat Herr Pastor Frommann, der vieljührige Freund des Jubilars, dem er auch zur Seite sass, um Gehör für einige Worte, die den grössten Anklang fanden. Er meinte unter Anderm, dass er freilich der einzige Laie in der Gescllschaft sei, aber eigentlich es dennoch nicht sei, weil die Theologie auch ihren Apparatus medicamentorum habe, der die Mittel der Pharmacie bei den Kranken of't recht unterstütze. - $\mathrm{Hr}$. Trapp, Professor der Pharmacie an der K. med. chirurgischen Akademic, hielt eine lateinische Ansprache, die sich in Variationen über Diligentia, Probitas und Benignitas des Jubilars recht sinnreich aussprach; wie überhaupt dieser unser liebenswürdiger Freund durch mehrere späterhin folgende joviale Trinksprüche viel zum allgemeinen Frobsinn der Gesellschaft beitrug. - IIr. Apoth. Gauger, Mitglied des Medicinalraths, hielt eine Anrede an den Jubilar, die namentlich dessen 'Thätigkeit und Fleiss besprach, als gedeihende Stiitzen jedes gewerblichen Geschäftes. - Unter l3egleitung der ausgesuchtesten Opernstücke und dem Klirren der Champagnerglïser wurde noch mancher heitere Spruch vernommen, an alle Anwesende das Portrait des Jubilars vertheilt und die gemüthliche Unterhaltung nach aufgehobener Tafel noch lange fortgesetzt.

Wir können die Erinnerung an die angenehm verlebten Stunden bei dieser Jubelfeier nicht schliessen, ohne den herzlichen Wunsch auszuduüeken, dass es recht, recht vielen Mitgliedern der so ehrenwerthen pharmaceutischen Corporation St. P'etersburgs von der gütigen Vorsehung bestimmt sein möge, nach einem 50jührigen thätigeu, rechtschaffenen Berufsleben, gleich Sr. Excellenz dem Herrn Apotheker $\mathrm{K}$ ä m merer, cinen solchen wohlverdienten Jubeltag zu feiern!

Dr. Maximilian Heine, Ehrenmitglied der pharmaceutischen Gesellschaft.

\section{Vereins - Angelegenheiten.}

\section{Bericht iiber die an 23. Juli d. J. abgehaltene Kreis- versammlung zu C'refeld.}

Seitens der Kreisdirectoren Richter, Biegmann und Dr. Schlienkanı waren die Mitglieder der betreffenden Kreise, so wie die Collegen überhaupt durch die Cöln. Zeitung zu einer Versammlung eingeladen.

Anwesend waren die Collegen: Richter, Kühtze, Kreitz und Roffhack aus Crefeld, Dr. Bausch, Westphal und Schlienkamp aus Düsseldorf, Feldha us ans Neuss, Riedel aus Rheydt, Becker aus IIüls, Menne aus Mühlheim, Le ucken aus Süchteln, Hintze aus Duisburg; ausserdem erfyeute der Herr Dr. Nauck, Director der Gewerbeschule, eingeladen vom Hrn. Collegen Richter, die Versammlung durch seine 'Theilnahme.

Kreisdirector Ri chter cröft'nete die Sitzung, begrüsste mit berzlichen Worten die Versammlung und sprach die Hoffnung aus, dass 